\title{
Relación entre las reservas de hierro maternas y del recién nacido
}

\author{
María Eugenia Vásquez-Molina, Q B.P., (1) Martha Corral-Terrazas, M. en C., ${ }^{(2)}$ \\ María Angélica A pezteguia, Q .B.P., ${ }^{(3)}$ Jorge $C$ armona-Sawasky, ${ }^{(2-4)}$ \\ Margarita Levario-Carrillo, M. en C. . $^{(2)}$
}

\section{Vásquez-Molina ME, Corral-Terrazas M, Apezteguia MA, Carmona-Sawasky J, Levario-Carrillo M. \\ Relación entre las reservas \\ de hierro maternas y del recién nacido. Salud Publica Mex 2001;43:402-407.} El texto completo en inglés de este artículo está disponible en: http://www.insp.mx/salud/index.html

\section{Resumen}

Objetivo. Determinar la relación de los valores hematoló gicos maternos y neo natales en casos de reservas maternas de hierro bajas, moderadas y normales. Material y métodos. Diseño transversal en el que se incluyó a 163 mujeres embarazadas y sus neonatos de término, derechohabientes del Hospital de Ginecología y 0 bstetricia número 15 del Instituto Mexicano del Seguro Social (IMSS) en Chihuahua, Chih., México. Se analizaron antecedentes maternos. Se determinaron niveles de hemoglobina, hematocrito y ferritina sérica en muestras maternas y de cordón umbilical. Se definieron reservas de hierro maternas de acuerdo a ferritina ( $\mu \mathrm{g} / \mathrm{l})$ : bajas $\leq 11.9$, moderadas de 12 a 20 y normales $\geq 20.1$. Se utilizó la prueba de Kruskal $W$ allis para establecer diferencias entre grupos, ji cuadrada para diferencia de proporciones y $r$ de Pearson para establecer la relación entre reservas de hierro maternas y del recién nacido. Resultados. Se determinó una débil correlación entre la ferritina materna y neonatal $r=0.14(p=0.07)$. Las medias geométricas de ferritina neonatal respecto a las reservas maternas bajas, moderadas y normales fue de 4.77, 4.85 y 5.02 , respectivamente $(p=0.12)$. Las reservas de hie-

\author{
Vásquez-Molina ME, Corral-Terrazas M, \\ Apezteguia MA, Carmona-Sawasky J, \\ Levario-Carrillo $M$. \\ Relationship between maternal \\ and neonatal iron stores. \\ Salud Publica Mex 2001;43:402-407. \\ The English version of this paper \\ is available at: http://www.insp.mx/salud/index.html
}

\begin{abstract}
A bstract
Objective.To establish the relationship of normal, low, and moderate blood iron values in mothers and their newborns. Material and Methods A cross-sectional study was conducted among 163 pregnant women and their newborns, users of Hospital de Ginecología y 0 bstetricia número 15, Instituto Mexicano del Seguro Social, from C hihuahua, $\mathrm{Me}$ xico. The mothers' clinical histories were collected and analyzed; hemoglobin, hematocrit, and ferritin serum levels were measured in maternal and umbilical cord samples. Iron maternal stores were determined by ferritin $(\mu \mathrm{g} / \mathrm{l})$ values as follows: low: $\leq 11$; moderate: 12-20; and normal: $\geq 20.1$. The Kruskal- Wallis test was used to establish differences among group; the chi-squared test to determine differences of proportions; and Pearson's correlation coefficient for assessing the association between maternal and newborn iron stores. Results. A weak correlation between maternal and neonatal ferritin was found $(r=0.14, p=0.07)$. G eometric means of neonatal ferritin for low, moderate, and normal maternal iron stores were $4.77,4.85$, and 5.02 respectively $(p=0.12)$. The maternal iron stores changed after iron supplementation $(p=0.01)$. Conclusions Iron stores in moth-
\end{abstract}

Proyecto financiado por la Coordinación de Investigación Médica del Instituto Mexicano del Seguro Social. Número de proyecto:FP - 0038 / 807.

(1) Laboratorio de Análisis Clínicos de la Unidad de Medicina Familiar N úmero 44 del Instituto Mexicano del Seguro Social, Chihuahua, México.

(2) Unidad de Investigación Médica en Epidemiología C línica, H ospital de G inecología y 0 bstetricia N 0.15, Instituto Mexicano del Seguro Social, C hihuahua, México.

(3) Laboratorio de Análisis Clínicos de la Clínica del Parque, Chihuahua, Chih., México.

(4) Estudiante de la Facultad de Medicina de la Universidad Autónoma de Chihuahua, México.

Fecha de recibido: 9 de agosto de 2000 - Fecha de aprobado: 13 de marzo de 2001

Solicitud de sobretiros: Q . B. P. María EugeniaVásquez Molina. Laboratorio de Análisis Clínicos de la Unidad de Medicina Familiar 44 del Instituto Mexicano del Seguro Social.Avenida Tecnológico y C. Pino S/N colonia Granjas Chihuahua, 31160 Chihuahua, México.

Correo Electrónico: eugenia@ megalink.net.mx. 
rro maternas se modificaron con el suplemento de hierro $(p=0.01)$. Conclusiones. Las reservas de hierro en el recién nacido están en estrecha relación con las maternas. Las mujeres que toman suplementos con hierro durante la gestación tienen reservas mayores al final del embarazo. El texto completo en inglés de este artículo está disponible en: http://www.insp.mx/salud/index.html

Palabras clave: reservas de hierro; ferritina; embarazo; recién nacido; México ers and their newborns are closely related. Women who take iron supplements during pregnancy have significantly higher iron stores at the end of pregnancy.The English version of this paper is available at: http://www.insp.mx/salud/ index.html

Key words: iron status; ferritin; pregnancy newborn; Mexico
L a anemia por deficiencia de hierro es uno de los problemas nutricios de mayor magnitud en el mundo. ${ }^{1-3}$ Se estima que alrededor de 2000 millones de personas sufren esta deficiencia y que más de la mitad está anémica; la prevalencia de anemia entre las mujeres embarazadas, los infantes y los menores de dos años de edad en los países en desarrollo supera el $50 \%$. La prevalencia de anemia por deficiencia de hierro en México, en mujeres embarazadas, es $18 \%{ }^{4}$ En maternas con anemia se han documentado consecuencias adversas inmediatas, como el retardo en el crecimiento intrauterino y cambios placentarios..$^{5-8}$ Existe controversia, para establecer los valores críticos de la deficiencia de hierro materna, que generan cambios en la reserva de hierro del recién nacido, y en cómo se afectan a largo plazo el crecimiento y el desarrollo del niño. ${ }^{9-12}$ En estudios que han relacionado el efecto de las reservas de hierro maternas sobre las reservas de hierro neonatales se muestra una alteración en ferritina, volumen globular medio (VCM) y hemoglobina corpuscular media $(\mathrm{HCM})^{13-14}$ cuando las reservas maternas son bajas. Por el contrario, se informa de ciertas investigaciones donde las reservas de hierro del recién nacido no se ven afectadas, aun cuando la condición materna no es adecuada. ${ }^{15-16}$

La determinación de ferritina en suero es empleada como una prueba accesible en la evaluación de la reserva de hierro corporal, ${ }^{17-19}$ aunque la cuantificación de receptores de transferrina es la medida que refleja de una mejor forma el estado funcional del hierro. ${ }^{20}$ Las reservas de hierro se han clasificado con base en los niveles de ferritina como bajas, moderadas y normales. ${ }^{13-21}$ La información sobre las alteraciones en los neonatos, cuando estas reservas son moderadas, es escasa.

Los objetivos del presente estudio son comparar los valores hematológicos de recién nacidos, hijos de madres con reservas bajas, moderadas y normales de hierro, y determinar la influencia de la suplementación con éste durante la gestación, en sus reservas maternas y neonatales.

\section{Material y métodos}

En el Hospital de Ginecología y Obstetricia No. 15 del Instituto Mexicano del Seguro Social, en Chihuahua, México, se diseñó un estudio transversal en el que se incluyeron 163 mujeres con diagnóstico de embarazo de 37 semanas o más, en trabajo de parto. El periodo de estudio fue de marzo de 1999 a marzo de 2000. El proyecto fue aprobado por el comité de ética. Al ingreso de la paciente a la sala de trabajo de parto, se realizó una entrevista para determinar las características generales, antecedente de tabaquismo durante la gestación, el peso pregestacional, la estatura y el índice de masa corporal (IMC) pregestacional, el cual se estratificó en bajo peso, pacientes con IMC menor a $19.8 \mathrm{~kg} / \mathrm{m}^{2}, 19.8-26 \mathrm{~kg} / \mathrm{m}^{2}$ peso normal, $26.1-29$ $\mathrm{kg} / \mathrm{m}^{2}$ sobrepeso y mayores a $29 \mathrm{~kg} / \mathrm{m}^{2}$, pacientes con obesidad. Se realizó un interrogatorio sobre los antecedentes obstétricos y la ingestión de sales ferrosas durante la gestación y se revisó el expediente clínico. A cada paciente se le explicó el objetivo del estudio, se le solicitó consentimiento informado por escrito y se le tomó una muestra de sangre venosa. Fueron excluidas las pacientes con alguna enfermedad como diabetes mellitus, preclampsia, eclampsia, o aquéllas con antecedente de transfusión sanguínea. Se consideró suplementación con hierro adecuada a la ingestión de sales ferrosas por un periodo igual o mayor a tres meses durante el periodo de gestación.

El pinzamiento del cordón umbilical se realizó entre 15 y 20 segundos después del parto; al momento del alumbramiento se tomó la muestra de sangre de la vena umbilical. Las muestras fueron procesadas de acuerdo a las siguientes técnicas: la hemoglobina, por la técnica de la cianometa hemoglobina, y el hematocrito, en tubo capilar y centrifugado durante cinco minutos.

La ferritina se determinó en suero por inmunoensayo, en el que se utiliza tecnología quimioluminométrica directa con reactivos de chiron diagnostics, con un coeficiente de variación intraensayo de $2.73 \%$. 
Las determinaciones se realizaron en un aparato ACS: 180, estandarizado respecto al material de referencia de la Organización Mundial de la salud (OMS) .

Se definieron reservas de hierro maternas como bajas, con valores de ferritina $\leq 11.9 \mu \mathrm{g} / \mathrm{l}^{14,21}$ moderadas entre 12 y $20^{13} \mu \mathrm{g} / 1$ y normales, con $\geq 20.1 \mu \mathrm{g} / \mathrm{l}^{13}$ Para identificar el efecto del suplemento con hierro durante la gestación, sobre las reservas maternas y neonatales, se consideraron con reservas bajas a aquellas mujeres con ferritina sérica $\leq 11.9 \mu \mathrm{g} / \mathrm{l}$, y con reservas suficientes a aquellas con niveles $\geq 12 \mu \mathrm{g} / \mathrm{l}$.

Se realizó un análisis exploratorio y univariado para cada una de las variables. Con el fin de establecer las diferencias entre los grupos conformados de acuerdo a las reservas de hierro, para un factor se utilizó la prueba de Kruskal Wallis por tratarse de tres grupos independientes, con variable dependiente, sin distribución normal en las variables cuantitativas. Con la prueba de ji cuadrada se determinó la diferencia de proporciones en las variables categóricas. Se realizó la transformación logarítmica de la ferritina y se utilizó la $r$ de Pearson para establecer la relación entre las reservas de hierro maternas y las del recién nacido. La influencia del tratamiento con suplemento de sales ferrosas, en las reservas de hierro de la madre y del neonato, se exploró con la $t$ de Student. Para la significancia se tomó el valor de $p<0.05$.

\section{Resultados}

Las características clínicas y demográficas del grupo estudiado, respecto a las reservas de hierro, se observan en el cuadro I, donde se muestra que los grupos fueron similares. De las 163 pacientes del estudio fue necesario excluir a siete debido a que no fue posible procesar la muestra para ferritina sérica, por presencia de hemólisis en el suero; el análisis final está basado en $\mathrm{n}=156$. El $94 \%$ acudió a su control prenatal. De acuerdo al número de embarazos $46 \%$ fueron primigestas, $44 \%$ de 2-3 gestas y 10\% con cuatro o más gestas. El $8 \%$ de las pacientes fumó durante el embarazo. El 76\% tomó suplemento con hierro durante la gestación. De acuerdo a las reservas maternas se encontró que $49 \%$ cursó con bajas reservas, $24 \%$ con moderadas y $27 \%$ con reservas normales. La correlación de los valores hematológicos entre la madre y el recién nacido se presenta en el cuadro II. Los valores hematológicos del neonato respecto a las reservas de hierro maternas se muestran en el cuadro III; al efectuar la regresión lineal para la ferritina neonatal de acuerdo a las reservas maternas se obtuvieron los siguientes datos: los hijos de mujeres con bajas reservas de hierro cursaron con una ferritina sérica al nacimiento de $4.77 \pm 0.62$
Cuadro I

Características clínicas de las madres de acuerdo a sus Reservas de hierRo. Instituto MEXicano del Seguro Social, Chihuahua, México, 1999-2000

\begin{tabular}{|c|c|c|c|c|}
\hline \multirow[b]{2}{*}{$\begin{array}{l}\text { Variable } \\
\qquad n=76\end{array}$} & \multicolumn{3}{|c|}{ Reservas de hierro maternas } & \multirow[b]{2}{*}{ p } \\
\hline & $\begin{array}{l}\text { Bajas* } \\
n=38\end{array}$ & $\begin{array}{c}\text { Moderadas* } \\
n=42\end{array}$ & Normales* & \\
\hline Edad (años) & $24 \pm 5$ & $24 \pm 5$ & $25 \pm 5$ & 0.66 \\
\hline Peso (Kg) & $60 \pm 11$ & $61 \pm 12$ & $59 \pm 11$ & 0.65 \\
\hline Talla (cm) & $160 \pm 7$ & $162 \pm 5$ & $161 \pm 5$ & 0.25 \\
\hline Semanas de gestación & $39 \pm 1$ & $39 \pm 1$ & $39 \pm 1$ & 0.55 \\
\hline Intervalo intergenésico (meses) $)^{\ddagger}$ & $51 \pm 37$ & $68 \pm 32$ & $54 \pm 36$ & 0.1 \\
\hline IMC $\left(\mathrm{kg} / \mathrm{m}^{2}\right)^{\S}$ & & & & 0.87 \\
\hline$\leq 19.7$ & $16(22 \%)$ & $5(3 \%)$ & $9(22 \%)$ & \\
\hline $19.8-26$ & $40(55 \%)$ & $25(66 \%)$ & $24(59 \%)$ & \\
\hline $26.1-29$ & $7(10 \%)$ & $4(11 \%)$ & $5(12 \%)$ & \\
\hline$\geq 29.1$ & $10(14 \%)$ & $4(11 \%)$ & $3(7 \%)$ & \\
\hline \multicolumn{5}{|c|}{$\begin{array}{l}\text { * Promedio } \pm \text { desviación estándar } \\
\text { ₹ Se excluyen pacientes primigestas para este análisis } \\
\S \text { Índice de masa corporal pregestacional, se muestra la n y la proporción } \\
\text { para cada categoría }\end{array}$} \\
\hline
\end{tabular}

\section{Cuadro II \\ Correlación de los valores hematológicos maternos y neonatales. Instituto Mexicano del Seguro Social, Chinuahua, México, 1999-2000}

\begin{tabular}{|c|c|c|c|c|}
\hline Variable & Madre* & Recién nacido* & $r^{\ddagger}$ & $p$ \\
\hline Hemoglobina ( $\mathrm{g} / \mathrm{l}$ ) & $120 \pm 10$ & $150 \pm 10$ & 0.11 & 0.13 \\
\hline Hematocrito (\%) & $39 \pm 3$ & $50 \pm 5$ & 0.09 & 0.22 \\
\hline Ferritina $(\mu \mathrm{g} / /)^{\S}$ & $2.6 \pm .69$ & $4.86 \pm .62$ & 0.14 & 0.07 \\
\hline
\end{tabular}

$\mu \mathrm{g} / 1(\beta=-29 p=0.06)$, los de mujeres con reservas moderadas $4.85 \pm 0.73 \mu \mathrm{g} / 1(\beta=-10 p=0.57)$, y aquellos recién nacidos de mujeres con reservas adecuadas de hierro $5.02 \pm 0.5 \mu \mathrm{g} / \mathrm{l}$.

No se encontraron diferencias en los niveles de ferritina sérica al nacimiento por género en recién nacidos femeninos $(\mathrm{n}=76)$; la media geométrica fue de $4.88 \pm 0.67 \mu \mathrm{g} / \mathrm{l}$ y en recién nacidos masculinos $(\mathrm{n}=80)$ de $4.84 \pm 0.58 \mu \mathrm{g} / 1$ ( $p=0.68)$.

El efecto de las sales ferrosas sobre las reservas maternas y del neonato se observa en la figura 1 , don- 


\section{Cuadro III \\ Valores hematológicos neonatales de ACUERDo A LAS RESERVAS DE HIERRO MATERNAS. Instituto Mexicano del Seguro Social, Chinuahua, México, 1999-2000}

\begin{tabular}{lcccc} 
& \multicolumn{3}{c}{ Reservas de hierro maternas } & \\
\cline { 2 - 4 } Variable neonatal & Bajas* & Moderadas* & Normales* & $p$ \\
Hemoglobina $(\mathrm{g} / \mathrm{l})$ & $150 \pm 10$ & $150 \pm 10$ & $150 \pm 10$ & 0.85 \\
\hline Hematocrito $(\%)$ & $50 \pm 5$ & $50 \pm 6$ & $49 \pm 4$ & 0.11 \\
\hline Ferritina $(\mu \mathrm{g} / \mathrm{l})^{\ddagger}$ & $4.77 \pm .62$ & $4.85 \pm .73$ & $5.02 \pm .50$ & 0.12 \\
& & & \\
* Promedio \pm desviación estándar & & \\
‡ N iveles de ferritina en escala logarítmica &
\end{tabular}

de el efecto positivo de la suplementación fue sobre los valores hematológicos maternos. Las mujeres que recibieron suplemento con hierro cursaron con niveles séricos de ferritina de $2.67 \pm 0.71$, mientras que en aquellas sin suplemento los niveles de ferritina fueron $2.37 \pm 0.57(p=0.02)$. La hemoglobina en el primer grupo fue de 12.1 \pm 1 , y en el segundo de $11.5 \pm 1(p<0.01)$. En el neonato la diferencia en los valores séricos de ferritina no fue significativa $(p=0.67)$.

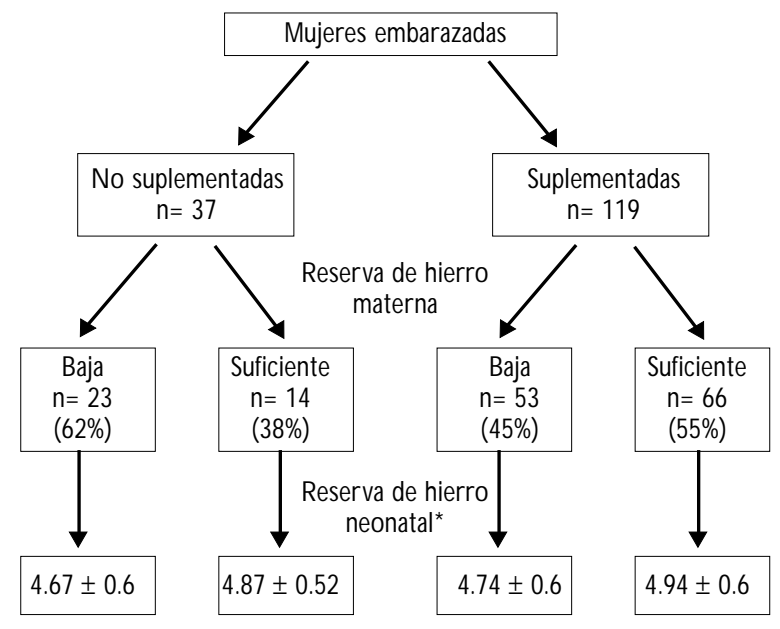

* N iveles de ferritina: media geométrica土desviación estándar, unidades $\mu \mathrm{g} / \mathrm{l}$

Figura 1. Reservas de hierro maternas y neonatales DE ACUERDO A LA INGESTIÓN DE SALES FERROSAS DURANTE la gestación. Instituto Mexicano del Seguro Social, Chinuahua, México, 1999-2000

\section{Discusión}

En este estudio se encontró deficiencia de hierro en 51\% de las mujeres embarazadas; de ellas, $27 \%$ corresponde a reservas de hierro bajas y $24 \%$ a reservas moderadas. Estos resultados son similares a los referidos para países en desarrollo, ${ }^{3-5}$ y mayores para países desarrollados. ${ }^{3-7}$ La correlación de los valores de hemoglobina y hematocrito maternos y neonatales no fue significativa, lo cual apoya lo informado en otros trabajos, ${ }^{15}$ ya que estos valores no se alteran hasta que las reservas de hierro están depletadas.

Una débil correlación entre los niveles de ferritina sérica materna y neonatal $(r=0.14)$ fue determinada $\mathrm{y}$ este resultado es similar a lo informado por otros autores: ${ }^{22-24}$ Nemet y colaboradores ${ }^{22}$ refieren una $r=0.15$ en una muestra de 218 binomios madre y recién nacido. En el estudio de Rusia y colaboradores, ${ }^{23}$ con una prevalencia de anemia por deficiencia de hierro de $34 \%$, se halló una $r=0.40(p<0.01)$.

En México, Baptista y colaboradores ${ }^{13}$ han determinado una diferencia significativa en la ferritina de neonatos cuyas madres cursaron con niveles de ferritina sérica $<10 \mu \mathrm{g} / \mathrm{l}$, con respecto a los recién nacidos de mujeres con cifras mayores, demostrando el efecto desfavorable sobre la reserva fetal de hierro en hijos de mujeres con ferropenia extrema.

En este estudio los niveles de ferritina sérica en neonatos no mostraron una diferencia con significancia estadística, aunque se puede apreciar una tendencia a incrementarse la ferritina neonatal con mayores niveles de ferritina materna.

En otros trabajos, ${ }^{15,25}$ la relación entre las reservas de hierro maternas y neonatales no han mostrado una asociación.

Lao y colaboradores, ${ }^{15}$ en un estudio de cohorte con una $\mathrm{n}=96$, informan una $r=0.10$, sugiriendo que el feto toma hierro de la madre independientemente de la reserva materna; sin embargo, las pacientes incluidas fueron evaluadas antes de la $20^{\mathrm{a}}$ semana de gestación y el 100\% recibió suplementos con hierro, lo cual pudiera influir en que los niveles de ferritina maternas no presentaran diferencias marcadas al final de la gestación, y así no podrían establecerse estratos de pacientes con bajos, moderados o adecuados niveles de ferritina en este periodo.

Los niveles de ferritina sérica no fueron diferentes en neonatos de acuerdo al género, como lo informado por Tamura y colaboradores ${ }^{26}$ quienes refieren mayores niveles de ferritina en recién nacidas. Los resultados son similares a los de Widemann y Jonetz-Mentzel; estos autores, al establecer valores de referencia, sólo encuentran diferencias en género en el grupo de 16 a 
18 años, hallando así niveles de ferritina mayores en varones. ${ }^{27}$

La suplementación con sales ferrosas se reflejó tanto en las reservas de hierro maternas como en la hemoglobina; en los recién nacidos se observó que los valores de ferritina fueron mayores en hijos de madres suplementadas con sales ferrosas, aunque la diferencia no fue significativa. Resultados similares fueron observados por Preziosi y colaboradores, ${ }_{1}^{14}$ quienes dieron seguimiento a niños, desde recién nacidos hasta los seis meses de edad, repartidos en dos grupos: uno, donde la madre e hijo fueron suplementados, y otro donde ninguno recibió suplementación. En los neonatos hijos de madres tratadas con suplemento de hierro se observó una mayor proporción de reservas al nacimiento, aunque no fue significativa; posteriormente, a los tres meses de edad, el consumo de sales ferrosas modificó significativamente las reservas de hierro en estos niños.

Resultados adversos en la salud perinatal también se presentan cuando los niveles de ferritina materna se encuentran elevados. ${ }^{28-29}$ En el estudio de Goldenberg y colaboradores ${ }^{17}$ se informó una razón de momios de 2.7 para parto pretérmino y 2.9 para bajo peso al nacer, en mujeres con niveles de ferritina sérica elevados.

Entre las limitaciones de este estudio se considera el hecho de que no se hizo la diferenciación con otras causas de anemia, al no determinar ácido fólico y vitamina B12. Por otro lado, el diseño sólo permite hablar de la correlación entre las reservas maternas y neonatales al final de la gestación.

En conclusión, estos resultados sugieren que las reservas de hierro de los recién nacidos se encuentran estrechamente relacionadas con las reservas de hierro maternas, y una evaluación de las reservas maternas de hierro al inicio de la gestación permitiría, por las implicaciones de esta condición, determinar a quién suministrar suplementos con hierro y contar con más probabilidades de disminuir los efectos adversos de la deficiencia de este metal en el recién nacido durante el desarrollo embrionario y fetal, así como una asociación con un mayor riesgo de enfermedades cardiovasculares en la vida adulta. ${ }^{30-31}$

\section{Agradecimientos}

Agradecemos el apoyo en el desarrollo de este trabajo al personal de Ginecología y Obstetricia No. 15 del Instituto Mexicano del Seguro Social en Chihuahua, Chihuahua, México.

\section{Referencias}

1. Mejía-D omínguez AM. Anemia en niños: Etiopatogenia y clasificación. Anemia por deficiencia de hierro. Bol Med Hosp Infant Mex 1997;54: 209-213.

2. Shine JW. Microcytic anemia.Am Fam Physician 1997;55:2455-2462.

3. Freire W B. La anemia por deficiencia de hierro: estrategias de la O PS/ O MS para combatirla. Salud Publica Mex 1998;40:199-205.

4. Martínez H, González-C ossio T, Flores M, Rivera-D ommarco J.A nemia en mujeres de edad reproductiva. Resultados de una encuesta probabilística nacional. Salud Publica Mex 1995;37:108-119.

5. Sarin AR. Severe anemia of pregnancy, recent experience. Int J 0 bstet Gynecol 1995:50 (Suppl 2):S45-S49.

6. 0 livares M, Llaguno S, Marín V, Hertrampf E, Mena P, Milad M. Iron status in low-birth-weight infants, small and appropriate for gestational age a follow-up study. Acta Paedriatr 1992;81:824-828.

7. ShollTO, Hediger ML, Fischer RL, Shearer JW. Anemia vs. Iron deficiency: Increased risk of preterm delivery in a prospective study. Am J Clin N utr 1992;55:982-988.

8. Reshstnikova OS, Burton GJ,Teleshova OV. Placental histomorphometry and morphometric diffusing capacity of the villous membrane in pregnancies complicated by maternal iron-deficiency anemia. Am J 0 bstet Gynecol 1995;173:724-727.

9. Chávez A, Martínez H, Guarneros N, Allen L, Pelton G. Nutrición y desarrollo psicomotor durante el primer semestre de vida. Salud Publica Mex 1998:40:111-118.

10. Baptista-González HA, Rosenfeld-Mann F, Ramírez-Maya A, Peñuela0 laya A. Cambios en los valores de hemoglobina y reserva de hierro en los primeros dos meses de vida. Bol Med Hosp Infant Mex 1999;56: 375-380.

11. Bello-G onzález A. D eficiencia de hierro: repercusión en el desarrollo encefálico y en el sistema inmunológico. Rev Mex Pue Ped 1997;5:91-94. 12. Inder TE, Clemett RS, Austin NC, Graham P, Darlow BA. High iron status in very low birth weight infants is associated with an increased risk of retinopathy of prematurity.J Pediatr 1997;131:541-544.

13. Baptista-González HA, Rosenfeld-Mann F, Mejía-López MD, Peñuela0 laya MA, Ruiz-Mares LO. Evaluación de la reserva materno fetal de hierro en embarazadas a término. Bol Med Hosp Infant Mex 1998:55:125-129. 14. Preziosi P, Prual A, Galan P, D auoda H, Boureima H, Hercberg S. Effect of iron supplementation on the iron status of pregnant women: consequences for newborns. Am J C lin N utr 1997;66:1178-1182.

15. Lao TT, Loong EPL, C hin RKH, Lam CW K, Lam YM. Relationship between newborn and maternal iron status and haematological indices. Biol N eonate 1991;60:303-307.

16.W hittaker PG, Macphail S, Lind T. Serial hematologic changes and pregnancy outcome. 0 bstetrics \& Gynecology 1996;88:33-39.

17. Goldenberg RL,TamuraT, D ubard M, Kelley E, Johnston BS, Copper RL et al. Plasma ferritin and prenancy outcome. Am J O bstet Gynecol 1996; 175:1356-1359.

18. Dézier JF,Vernet M. Détermination de la ferritine sérique. Intéret et limites. Presse Med 1992;21:1283-1286.

19. Kar-Fai T, Lao TT. Hemoglobin and red cell indices correlated with serum ferritin concentration in late pregnancy. 0 bstetrics \& G ynecology 1999:93:427-431.

20. Rusia U, Flowers C, Madan N, A garwal N, Sood SK, Sikka M. Serum transferring receptor levels in the evaluation of iron deficiency in the neonate. Acta Paediatr Jpn 1996;38:455-459.

21. Baptista-G onzález HA, Peñuela-O laya MA, N egrete-Valenzuela F,Vela J. Utilidad de los índices eritrocitarios en el estudio de la reserva de hierro del lactante menor. Bol Med Hosp Infant Mex 1993;50:639-644. 
22. N emet K, Andrassy K, Bognar K, C zappan P, Stuber A, Simonovits I. Relationship between maternal and infant iron stores: 1 full term infants. Haematolgia (Budap) 1986;19:197-205.

23. Rusia U. Madan N, A garwal N, Sikka M, Sood SK. Effect of maternal iron deficiency anaemia on foetal outcome. Indian J Pathol Microbiol 1995;38:273-279.

24. MacPhail AP, Charlton RW, Bothwell TH, Torrance JD. The relationship between maternal and infant iron status. Scan J Haematol 1980;25:141150.

25. Pavelka R, Kofler E. Linkesch W, Pollak A. Serum ferritin in pregnancy at term and newborn. Pediatr Padol 1981;16:443-450.

26. Tamura T, H ou J, G oldenberg RL, Johnston KE, C liver SP. Gender difference in cord serum ferritin concentrations. Biol Neonate 1999;75: 343-349.

27. W iedemann $G$, Jonetz-Mentzel L. Establishment of reference ranges for ferritin in neonates, infants, children and adolescents. Eur J C lin Chem Biochem 1993;31:453-457.
28. Lao TT,Tam KF, Chan LY.Third trimester iron status and pregnanacy outcome in non - anaemic women; pregnancy unfavorably affected by maternal iron excess. Human Reproduction 2000;15:1843-1848.

29. Fellman V, Rapola J, Pihko H, Varilio T, Raivio KO Iron-overload disease in infants involving fetal growth retardation, lactic acidosis, liver haemosiderosis, and aminoaciduria. Lancet 1998;351:490-493.

30. Barker D PJ, Gluckman PD, Godfrey KM, Harding JE, O wens JA, Robinson JS. Fetal nutrition and cardiovascular disease in adult life. Lancet 1993;34:938-941.

31. Thame M, 0 smond C, W ilks RJ, Bennett Fl, McFarlane-Anderson N, Forrester TE. Blood Pressure is Related to Placental Volume and Birth W eight. Hypertension 2000;35:662-667. 